\title{
Discovery of effective solvents for platelet graphite nanofibers
}

L. Guardia $^{1 *}$, J.I. Paredes ${ }^{1}$, S. Villar-Rodil ${ }^{1}$, J-N. Rouzaud ${ }^{2}$, A. Martínez-Alonso ${ }^{1}$, J.M.D. Tascón ${ }^{1}$

${ }^{1}$ Instituto Nacional del Carbón, INCAR-CSIC, Apartado 73, 33080 Oviedo, Spain

${ }^{2}$ Laboratoire de Géologie de l'Ecole Normale Supérieure, UMR CNRS 8538, 24 rue

Lhomond, 75321 Paris Cedex 5, France

\begin{abstract}
The dispersibility of platelet-type graphite nanofibers (PGNFs), an archetype of carbon material with a surface dominated by graphitic edge planes, has been measured in 28 solvents and rationalized on the basis of solvent surface tension and Hansen solubility parameters. Successful solvents possess surface tensions of $\sim 25-35 \mathrm{~mJ} \mathrm{~m} \mathrm{~m}^{-2}$ and substantial values of the hydrogen-bonding Hansen parameter $\left(\delta_{\mathrm{H}} \sim 14-16 \mathrm{MPa}^{1 / 2}\right)$, and many of them are alcohols, such as 1-butanol, ethanol or cyclohexanol. Such result is mainly attributed to the fact that the PGNF edge planes are decorated with oxygen functional groups. The dispersion behavior of the nanofibers could be changed to that typically exhibited by carbon nanotubes and graphene by means of a high temperature annealing that converted their surface edge planes to curved basal planes.
\end{abstract}

\section{Introduction}

The ability to manipulate and process nanostructured materials in the liquid phase as stable colloidal dispersions constitutes an important and desirable feature that facilitates both their implementation in many prospective technological applications and their use

\footnotetext{
* Corresponding author: Telephone number: (+34) 9851190 90. Fax number: (+34) 9852976 62. E-mail address: lauraguardia@incar.csic.es (L.Guardia)
} 
in fundamental research [1,2]. This is true in particular for nanostructured carbon materials, and most notably in the case of carbon nanotubes and graphene, which are widely investigated on account of their superior electrical, mechanical, thermal and optical properties [3-8]. For example, the availability of colloidal dispersions of carbon nanotubes and graphene has allowed the preparation of thin films, coatings or composite materials towards different potential uses [8-10].

Although nanostructured carbons can be efficiently dispersed in aqueous and organic media with the aid of suitable stabilizers such as surfactants, polymers or biomolecules [3,4,6,11-13], the use of the latter is not always advantageous: stabilizers are subsequently difficult to remove completely and their presence may degrade the performance of materials and devices prepared from such dispersions [14]. Hence, in many instances direct dispersion of the nanostructured carbon in the solvent alone would be preferable. To that effect, work carried out over the last years has determined that both graphene (pristine as well as in chemically modified form) [15-19] and carbon nanotubes [20-24] are amenable to form stable colloids only in certain solvents. Such behavior has been rationalized in terms of the energetic cost of exfoliating and dispersing the starting material (e.g., graphite or carbon nanotube bundles) in the solvent, which in turn can be estimated on the basis of either the cohesive energy density (i.e., using Hildebrand and Hansen solubility parameters) or, alternatively, the surface energy of both solvent and nanomaterial [16,22-24]. For example, Coleman and co-workers have shown that successful solvents for pristine graphene and carbon nanotubes are characterized by surface energies that match or are close to that of the basal plane of graphite $\left(\sim 70 \mathrm{~mJ} \mathrm{~m} \mathrm{~m}^{-2}\right.$, corresponding to solvent surface tensions of $\sim 40$

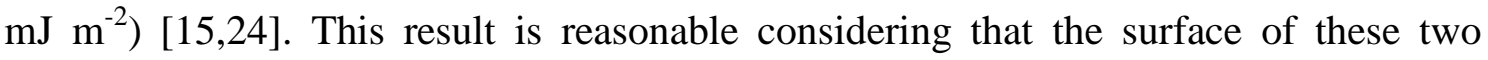


nanomaterials is essentially a graphitic basal plane and therefore the solvent molecules are effectively interacting with such a type of carbon surface.

However, graphitic carbon materials are well known for their diversity and not all of them are dominated by basal structures surface-wise [25-27]. In this regard, the most prominent example is probably that of platelet-type graphite nanofibers (PGNFs), which are made up of uniform-sized graphene layers (typically 40-200 nm) stacked along the nanofiber axis with lengths up to a few micrometers [28-30]. Even though PGNFs possess a rather crystalline structure, their surface is essentially constituted by graphitic edge planes rather than basal planes, the latter being oriented approximately perpendicular to the nanofiber surface instead. This particular surface conformation can be expected to make a difference in the dispersion behavior of PGNFs in solvents compared to that of other graphitic nanomaterials with a surface comprised of basal planes (e.g., graphene and carbon nanotubes). From a fundamental science perspective, such issue is therefore worth investigating, but to the best of our knowledge it has not yet been addressed. From a practical point of view, we note that PGNFs are mainly employed as a metal nanoparticle (e.g., Pt, $\mathrm{Pd}$ or $\mathrm{Ru}$ ) support for use in catalysis and electrocatalysis. While defects must be introduced in the inert, basal plane-structured surface of other carbonaceous supports to create anchoring sites, the edge planestructured surface of PGNFs directly provides a large number of anchoring sites allowing strong interaction with the metal clusters and molecules [29,31-36]. This Preparation of PGNF-supported metal catalysts is frequently carried out in the liquid phase (e.g., in toluene or ethylene glycol) [32,33,35,37]. Thus, a detailed knowledge of the dispersibility of this type of nanostructured carbon material in solvents could be useful in the design of improved preparation routes for such catalysts. 
We have investigated the dispersion behavior of PGNFs in a range of solvents, which we report here. The results are quantitatively analyzed on the basis of the Hildebrand and Hansen solubility parameters (cohesive energy density) and surface energy of the solvents, indicating clearly different trends in the dispersibility of these nanofibers compared to that of carbon nanotubes and graphene.

\section{Experimental}

The PGNFs, solvents and other reagents used in this work were acquired from Sigma-Aldrich. The nanofibers (99\% wt. carbon, $0.5-5 \mu \mathrm{m}$ in length and $50-250 \mathrm{~nm}$ in width by TEM, particle size: average width $\times$ average length: $100 \mathrm{~nm} \times 2.5 \mu \mathrm{m})$ were produced by a catalytic chemical vapor deposition process followed by an acid oxidation treatment to remove the metal catalyst. For reasons that will be commented upon below, both as-prepared and high temperature-annealed PGNFs were investigated. The latter were obtained by heat treatment of the as-prepared sample at $2400{ }^{\circ} \mathrm{C}$ for $1 \mathrm{~h}$ under Ar atmosphere.

Unlike the case of carbon nanotubes and graphene, dispersion of the PGNFs in the solvents did not require sonication. This is because the as-prepared powder is generally in a rather loose, non-agglomerated (only somewhat tangled) state, so a large amount of external mechanical energy, like that provided by ultrasound treatment, was not strictly necessary to extract individual nanofibers from the powder material. The dispersions could be prepared just by magnetically stirring the PGNF powder in the solvents, although a mild and brief ( $\leq 30 \mathrm{~min})$ sonication treatment was additionally implemented to undo some of the most intricately tangled objects and thus increase the amount of suspended material. However, prolonged sonication was not carried out to avoid an excessive cutting of the nanofibers, as this could lead to very short pieces with 
significant fractions of exposed basal planes, which in turn would be undesirable for the purpose of the present investigation. Thus, in a typical dispersion procedure, $50 \mathrm{mg}$ of PGNFs were added to $10 \mathrm{~mL}$ of solvent in a $25 \mathrm{~mL}$ vial and stirred with a magnetic bar at $350 \mathrm{rpm}$ for $2 \mathrm{~h}$. The suspension was then subjected to mild sonication in an ultrasound bath cleaner (J.P. Selecta Ultrasons system, $40 \mathrm{kHz}$ ) for $30 \mathrm{~min}$, centrifuged at $3000 \mathrm{~g}$ for $5 \mathrm{~min}$, and finally the top $\sim 75 \%$ of the resulting supernatant was collected for subsequent use. The amount of material suspended in the solvents was evaluated by

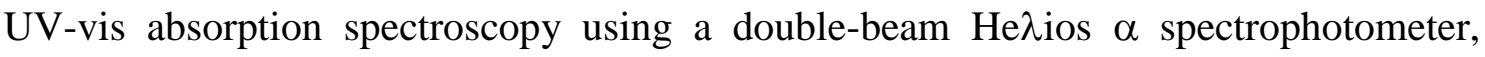
from Thermo Spectronic. More specifically, the absorbance per light path length (A/l) at a given wavelength $(660 \mathrm{~nm})$ was measured, which should be proportional to the dispersed concentration (C) according to the Lambert-Beer law: $\mathrm{A} / 1=\alpha \mathrm{C}$, where $\alpha$ is the extinction coefficient.

Characterization of the as-prepared PGNFs and heat-treated PGNFs (HT-PGNFs) was carried out by field-emission scanning electron microscopy (FE-SEM), energydispersive X-ray (EDX) spectroscopy, transmission electron microscopy (TEM), X-ray diffraction (XRD), Raman spectroscopy, thermogravimetric analysis (TGA) and X-ray photoelectron spectroscopy (XPS). FE-SEM images and EDX spectra were recorded on a Quanta FEG 650 apparatus (FEI Company) with samples prepared by depositing a small amount of the dry nanofiber powder onto the sample holder by means of doublesided carbon adhesive tape or, for samples dispersed in the liquid phase, by drop-casting the suspension onto a highly oriented pyrolytic graphite (HOPG) substrate. TEM imaging was accomplished either with a JEOL 2011 microscope equipped with a $\mathrm{LaB}_{6}$ gun and operating at $200 \mathrm{kV}$ or with a JEOL $2000 \mathrm{EX}-\mathrm{II}$ instrument operated at $160 \mathrm{kV}$. To this end, the dry nanofiber powder was first briefly sonicated in ethanol and then a few microliters of the resulting suspension were cast onto a copper grid (200 mesh) 
covered with a lacey amorphous carbon film and allowed to dry. XRD patterns were obtained on a Bruker D8 Advance diffractometer using $\mathrm{Cu} \mathrm{K}_{\alpha}$ radiation $(\lambda=0.154 \mathrm{~nm})$ at a step size and dwell time of $0.02^{\circ}(2 \theta)$ and $1 \mathrm{~s}$, respectively. Raman spectra were recorded on a Horiba Jobin-Yvon LabRam instrument using a laser with excitation wavelength of $532 \mathrm{~nm}$ and low incident power $(\sim 0.5 \mathrm{~mW})$ to avoid damage to the sample. TGA was performed by means of an SDT Q600 thermobalance (TA Instruments) under synthetic air flow $\left(100 \mathrm{~mL} \mathrm{~min}^{-1}\right)$ at a heating rate of $10{ }^{\circ} \mathrm{C} \mathrm{min}^{-1}$ and using Pt crucibles. XPS measurements were carried out on a SPECS spectrometer with a $\mathrm{Mg} \mathrm{K} \alpha \mathrm{X}$-ray source $(1253.6 \mathrm{eV}, 100 \mathrm{~W})$, working at a pressure of $10^{-7} \mathrm{~Pa}$. The specimens for XPS were prepared by pelletizing the nanofibers with a hydraulic press.

\section{Results and discussion}

\subsection{General characterization of the PGNFs}
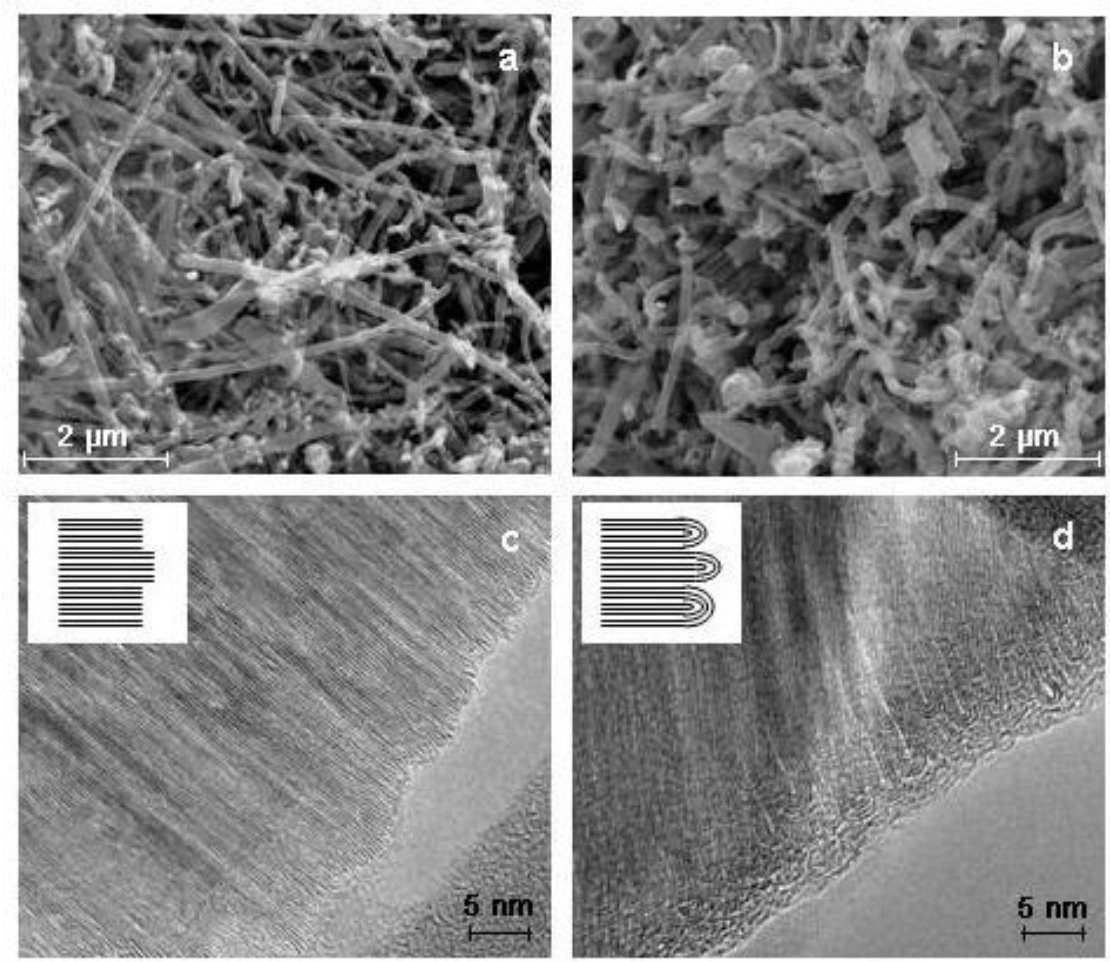
Fig. 1. Representative FE-SEM images of the as-prepared (a) and heat-treated (b) PGNFs. TEM images of the as-prepared (c) and heat-treated (d) nanofibers. Schematic representations of the PGNF surface structure are given as insets to (c) and (d).

Microscopic characterization (FE-SEM and TEM) of as-prepared PGNFs and HTPGNFs revealed that both consist of individual nanofibers with graphene layers stacked perpendicularly to the fiber axis (Fig. $1 \mathrm{a}$ and $1 \mathrm{~b}$ and images in the Supplementary content). However, their surface structure differs, being graphitic edge planes in the asprepared PGNFs (Fig. 1c) but closed loop, curved graphitic basal planes in the HTPGNFs (Fig. 1d), as previously reported [27,38-42]. This difference impacts their reactivity, as confirmed by TGA in air (Fig. 2a and 2b), and their structural disorder, as studied by Raman spectroscopy (Fig. 2c). Gasification starts at $\sim 550{ }^{\circ} \mathrm{C}$ (Fig. 2a) for the as-prepared PGNFs but at $\sim 700{ }^{\circ} \mathrm{C}$ for HT-PGNFs and graphite powder (Fig. 2b), temperatures characteristic, respectively, of the beginning of the etching of graphitic edge planes (and defects) [43], and of basal planes [43,44]. The ratio of the integrated intensity of the $\mathrm{D}\left(\sim 1350 \mathrm{~cm}^{-1}\right)$ and $\mathrm{G}\left(\sim 1582 \mathrm{~cm}^{-1}\right)$ bands $\left(\mathrm{I}_{\mathrm{D}} / \mathrm{I}_{\mathrm{G}}\right)$ in the Raman spectra (Fig. 2c), which correlates with structural disorder in graphitic materials $[45,46]$, notably diminishes upon high temperature treatment (from $\sim 1.9$ to $\sim 0.5$ ), due to closing of graphitic edges [38]. Additional Raman details can be found in the Supplementary content. Concerning XRD (Fig. 2d), a sharp and strong (002) peak at $\sim 26^{\circ}$ (20) was the main feature for both the PGNFs (black) and HT-PGNFs (gray), thus evidencing a high degree of order in the stacking of the graphene layers. The interlayer spacing $\left(\mathrm{d}_{002}\right)$ and stacking size $\left(\mathrm{L}_{\mathrm{c}}\right)$ values were, respectively, 0.337 and $\sim 17 \mathrm{~nm}$ for the PGNFs, and 0.336 and $\sim 25 \mathrm{~nm}$ for HT-PGNFs, indicating a slightly better crystallinity for the latter. 

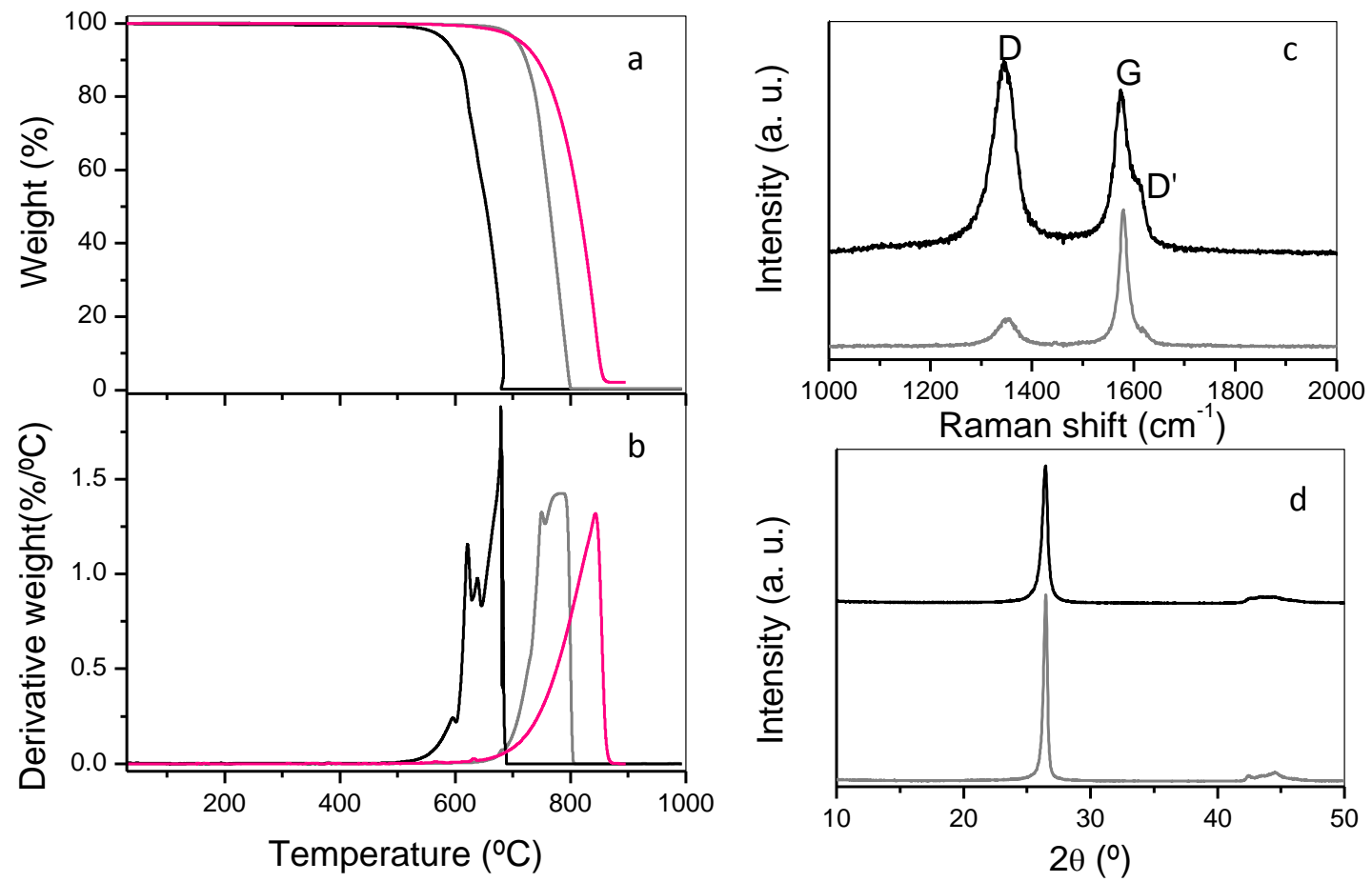

Fig. 2. Thermogravimetric (TG, panel a) and differential thermogravimetric (DTG, panel b) plots of the PGNFs (black) and HT-PGNFs (gray) and of a natural graphite powder (red). First-order Raman spectra (c) and XRD patterns (d) for the PGNFS (black) and HT-PGNFs (gray).

The surface chemistry characteristics of the nanofibers, which are expected to play an important role in their dispersion behavior in the liquid phase, were investigated by XPS (Fig. 3). It could be anticipated that the edge plane-structured surface of the asprepared PGNFs would be decorated with oxygen functionalities, as is typical for the exposed layer edges of graphitic materials $[47,48]$. Indeed, within the detection limit of this technique, only carbon and oxygen were found on the surface of both as-prepared and heat-treated samples (Fig. 3a). For the as-prepared PGNFs, the O/C atomic ratio was measured to be 0.024 , value from which it can be estimated that about $70 \%$ of the edge carbons that constitute the nanofiber surface are decorated with oxygen atoms (see Supplementary content for its derivation). As could be expected, the $\mathrm{O} / \mathrm{C}$ atomic ratio for HT-PGNFs is much smaller (0.006). Graphitization brings about a more 
homogeneous chemical environment for carbon, as confirmed by a narrower high resolution $\mathrm{C} 1 \mathrm{~s}$ band (Fig. 3b) and an increase in the electron delocalization, reflected in a more intense $\pi \rightarrow \pi^{*}$ band ( $291 \mathrm{eV}$, Fig. $\left.3 b\right)$ ). The oxygen functionalities in both samples are of the same kind (Fig. 3c), mainly single-bonded oxygen [49].

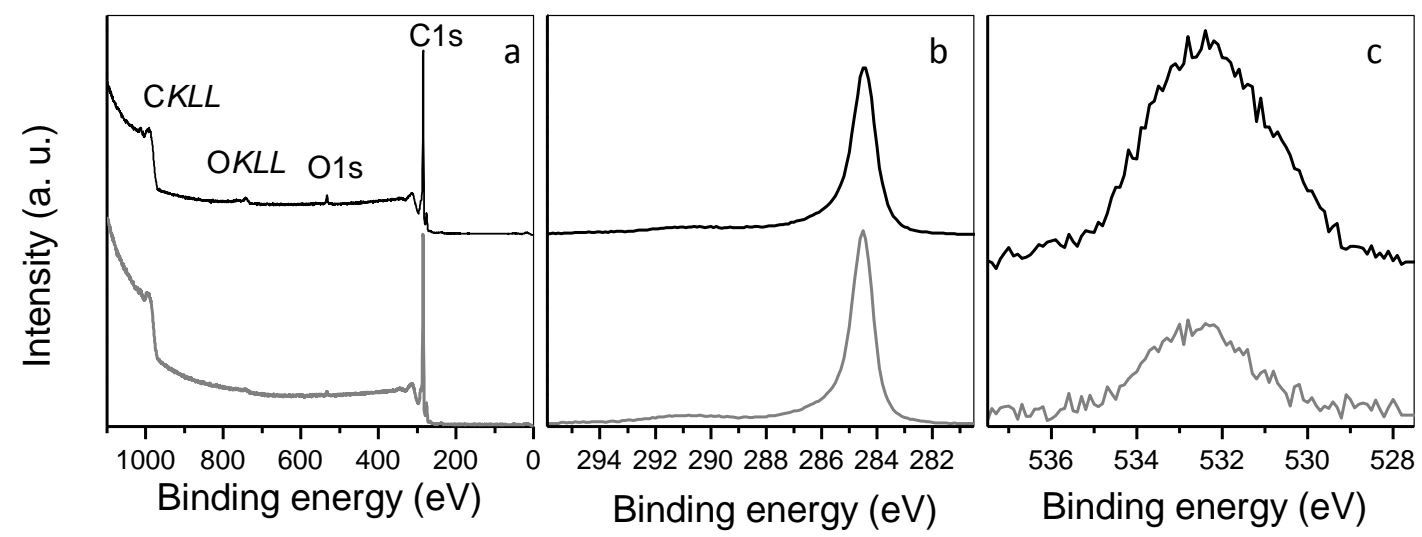

Fig. 3. X-ray photoelectron spectra recorded for the PGNFs (black) and HT-PGNFs (gray): survey spectra (a), and high resolution core level spectra for C1s (b) and O1s (c).

\subsection{Dispersion behavior of PGNFs in solvents}

The solubility of a given solute in solvents can be rationalized by the theory of Hansen solubility parameters, which is based on the analysis of the cohesive energy density of both components [50]. Although such a theory is best suited for molecular solutes, it has also been successfully applied to understand and predict the dispersion behavior in solvents and solvent mixtures of nanostructured materials (e.g., carbon nanotubes, graphene and several inorganic graphene analogues) [4,16,18,19,2224,51,52]. Nevertheless, for nanostructured materials Coleman and co-workers have proposed using the surface energy, rather than the cohesive energy density, as an alternative solubility parameter [4,24]. In principle, the dispersibility of a solute in a solvent is determined by the energetic cost of mixing the two of them, which in turn is given by their enthalpy of mixing. For a surface energy-based analysis of the PGNFs, 
their enthalpy of mixing per unit volume of solvent $\left(\Delta H_{m i x}\right)$ would be written to a first approximation as [4]

$$
\Delta H_{m i x}=\frac{4}{w}\left(\delta_{N F}-\delta_{s o l}\right)^{2} \phi
$$

where $w$ is the nanofiber width, $\phi$ is the volume fraction of PGNFs in the solvent and $\delta_{N F}=\sqrt{E_{\text {surf }}^{N F}}\left(\delta_{\text {sol }}=\sqrt{E_{\text {surf }}^{\text {sol }}}\right)$ is the square root of the surface energy of the nanofibers (solvent). Similarly, if we base the analysis on (total) cohesive energy densities, the enthalpy of mixing would take the form [4]

$$
\Delta H_{m i x}=\left(\delta_{T, N F}-\delta_{T, s o l}\right)^{2} \phi(1-\phi)
$$

where $\phi$ has the same meaning as in equation (1) and $\delta_{T, N F}\left(\delta_{T, s o l}\right)$ is the Hildebrand solubility parameter of the nanofibers (solvent). The Hildebrand solubility parameter of a system is given by the square root of its total cohesive energy density [50]. Equations (1) and (2) indicate that the energetic cost of dispersing the solute (PGNFs) in the solvent is minimized (and therefore the dispersed amount should be maximized) for solvents with surface energy and Hildebrand solubility parameter matching those of the solute.

To identify successful solvents for the as-prepared PGNFs, dispersions were prepared, as detailed in the Experimental section, by stirring, briefly sonicating and finally centrifuging the material in different solvents that cover a wide range of surface energies (or, equivalently, surface tensions). Fig. 4a shows a digital photograph of a representative series of the resulting dispersions arranged from left to right in increasing order of solvent surface tension. It was observed that the amount of suspended material is largest for solvents with surface tension roughly between 20 and $35 \mathrm{~mJ} \mathrm{~m}^{-2}$, and also that some of the most effective solvents are alcohols such as $n$-butanol or ethanol. The UV-vis absorption spectrum of a dispersion of the as-prepared PGNFs in $n$-butanol is 
presented in Fig. 4b, which exhibits an absorption peak located at $\sim 258 \mathrm{~nm}$ together with strong absorbance in the whole wavelength range above $258 \mathrm{~nm}$. These features are characteristic of carbon materials with a high degree of electronic conjugation (i.e., graphitic materials), including pristine graphene, highly reduced graphene oxide or multiwalled carbon nanotubes [12,53,54], and are therefore consistent with the presence of PGNFs suspended in the solvent. Such a point was directly corroborated by dropcasting the dispersions onto an HOPG substrate and imaging it by FE-SEM (Fig. 4c). Individual nanofibers $\sim 0.25-3 \mu \mathrm{m}$ in length, but typically below $1 \mu \mathrm{m}$, were seen to sparsely cover the HOPG substrate. This indicated that the dispersion procedure shortened the nanofibers to some extent, probably during the sonication step. In fact, when such step was omitted from the preparation of the dispersions, the suspended nanofibers tended to be somewhat longer, but were also frequently observed as entangled aggregates of several nanofibers. However, we also note that in all cases the dispersed objects retained their original nanofibrous morphology and were not exfoliated into thin graphitic platelets. As visualized by TEM, sonication did not either alter the microscopic structure of the nanofiber surface, which still consists of graphitic edge planes (images not shown).

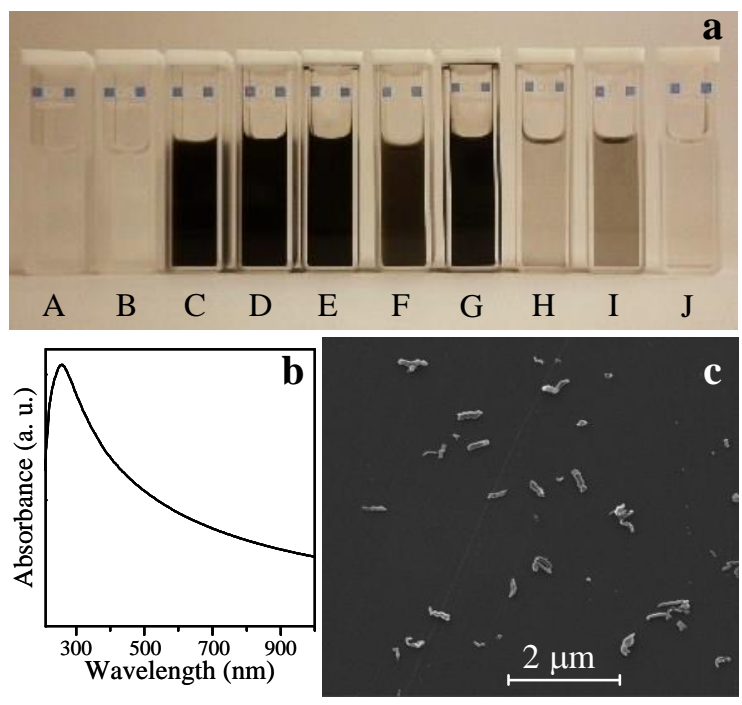


Fig. 4. (a) Digital photographs of cuvettes containing dispersions of as-prepared PGNFs in the following solvents (the bracketed numbers refer to their surface tension in $\mathrm{mJ} \mathrm{m}^{-}$ $\left.{ }^{2}\right)$. A: $n$-pentane (16.05); B: $n$-hexane (18.42); C: ethanol (22.32); D: 1-butanol (24.67); E: $o$-dichlorobenzene (26.84); F: acetonitrile (29.29); G: cyclopentanone (33.87); H: $\mathrm{N}, \mathrm{N}$-dimethylformamide (36.76); I: $\mathrm{N}$-cyclohexyl-2-pyrrolidone (38.8); J: $N$-methyl-2pyrrolidone (40.07). (b) UV-vis absorption spectrum of PGNF dispersion in 1-butanol. (c) FE-SEM image of a typical PGNF dispersion drop-cast onto HOPG.

To evaluate the dispersing ability of the different solvents in a more quantitative manner, we measured the absorbance of their corresponding suspensions at a given wavelength $(660 \mathrm{~nm})$, which can be expected to be proportional to the PGNF concentration in the solvent. The results are given in Table S1 of the Electronic Supplementary Information (ESI) for the 28 solvents employed, together with their surface tension and solubility parameters obtained from the literature $[50,55]$. On the basis of the results of Fig. 4a, many of the employed solvents were purposely chosen to have surface tensions in the $20-35 \mathrm{~mJ} \mathrm{~m}^{-2}$ range. The absorbance data are plotted in Fig. $5 \mathrm{a}$ and $\mathrm{b}$ versus solvent surface tension and Hildebrand solubility parameter, respectively. It can be noticed that the dispersed amount is maximum for solvents having values of these two parameters within a relatively narrow range, i.e. surface tensions approximately between 25 and $35 \mathrm{~mJ} \mathrm{~m}^{-2}$ (corresponding to surface energies of $\sim 55-65 \mathrm{~mJ} \mathrm{~m}^{-2}$ ) and Hildebrand parameters around $23 \mathrm{MPa}^{1 / 2}$. This result is consistent with what equations (1) and (2) generally predict and indicates that the surface energy and Hildebrand solubility parameter for the as-prepared PGNFs should also be around 55-65 $\mathrm{mJ} \mathrm{m}^{-2}$ and $23 \mathrm{MPa}^{1 / 2}$, respectively. In the case of carbon nanotubes and 
graphene, successful solvents have been reported to possess slightly larger surface energies of around $70 \mathrm{~mJ} \mathrm{~m}^{-2}$ (surface tensions $\sim 40 \mathrm{~mJ} \mathrm{~m}^{-2}$ ), which are close to the surface energy of the basal plane of graphite $[15,24]$. As opposed to carbon nanotubes and graphene, the surface of the as-prepared PGNFs is thought to be mainly constituted by graphitic edge planes rather than basal planes. Hence, successful solvents for these nanofibers would in principle be expected to exhibit surface energies close to that of the edge plane. Theoretically, the surface energy of an ideal graphitic edge plane should be much higher than that of the basal plane, i.e., it should be well above $70 \mathrm{~mJ} \mathrm{~m}^{-2}$ [56]. Nevertheless, the XPS measurements discussed previously suggested that most of the edge carbons forming the surface of the as-prepared PGNFs are stabilized by oxygen groups, which is the most common termination of layer edges in graphitic materials $[47,48]$, and therefore the surface energy of the nanofibers should be significantly lower than that of the ideal edge plane. Furthermore, on the basis of experimental measurements, Donnet et al. have documented that the surface energy of the oxidized prismatic (edge) plane of highly oriented pyrographite, which is the same type of surface structure as that of the as-prepared PGNFs, is somewhat smaller than the value determined by the same authors for the graphite basal plane ( 47 vs. $\left.62 \mathrm{~mJ} \mathrm{~m}^{-2}\right)$ [57]. The dispersion behavior of the as-prepared PGNFs reported here is in agreement with such result: successful solvents for the nanofibers possess surface energies slightly below those of the best solvents known to disperse nanostructured carbons with basal plane surfaces, such as carbon nanotubes and graphene ( $55-65$ vs. $\left.70 \mathrm{~mJ} \mathrm{~m}^{-2}\right)$.

We note, however, that the Hildebrand solubility parameters of solvents that disperse effectively the as-prepared PGNFs are very close to those of good carbon nanotube and graphene solvents $\left(\sim 23 \mathrm{MPa}^{1 / 2}\right)$, even though the specific solvents are very different in each case [16,24]. For instance, $N$-cyclohexyl-2-pyrrolidone, 
cyclopentanone and $N$-methyl-2-pyrrolidone are known to be very efficient solvents for carbon nanotubes and graphene, alcohols such as ethanol or isopropyl alcohol performing comparatively poorly, while for the as-prepared PGNFs the reverse is the case. This is because matching of the Hildebrand solubility parameters of solute and solvent [equation (2)] can be generally regarded as a necessary but not sufficient condition for successful dispersion of the solute in the solvent. Indeed, similar to what has been observed previously for the other carbon nanostructures [16,24], some solvents with Hildebrand parameters close to $23 \mathrm{MPa}^{1 / 2}$ could not successfully disperse the asprepared nanofibers (see Fig. 5b).
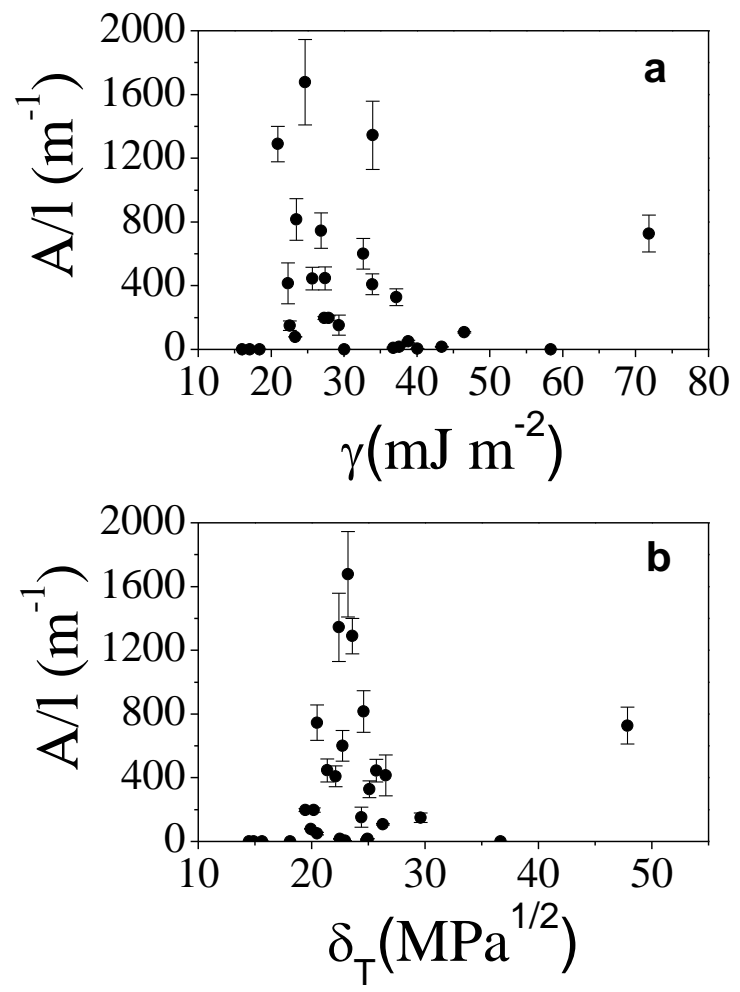

Fig. 5. Absorbance at $660 \mathrm{~nm}$ of PGNF dispersions in solvents plotted as a function of solvent surface tension (a) and Hildebrand solubility parameter (b).

To better discriminate the properties that make good PGNF and nanotube/graphene solvents to be different from each other, a Hansen solubility parameter analysis was carried out. In the framework of the theory of Hansen solubility parameters, the total 
cohesive energy density of a system is written as the sum of dispersive, polar and hydrogen-bonding contributions, so that the enthalpy of mixing for a solute(PGNF)/solvent pair is given by [51]

$$
\Delta H_{m i x}=\left\lfloor\left(\delta_{D, N F}-\delta_{D, s o l}\right)^{2}+\left(\delta_{P, N F}-\delta_{P, s o l}\right)^{2}+\left(\delta_{H, N F}-\delta_{H, s o l}\right)^{2}\right\rfloor \phi(1-\phi)
$$

where $\phi$ is the volume fraction of PGNFs in the solvent and $\delta_{i, N F}\left(\delta_{i, s o l}\right)$ is a Hansen solubility parameter of the nanofibers (solvent), with $i=\mathrm{D}, \mathrm{P}$ or $\mathrm{H}$ corresponding to the dispersive, polar and hydrogen-bonding parameters, respectively. The Hansen parameter $\delta_{i}$ is the square root of the $i$ component of the cohesive energy density. According to equation (3), the dispersibility of the nanofibers will be most favored in those solvents that have the three Hansen solubility parameters matching their respective nanofiber counterparts, and therefore the dispersed amount should be expected to peak for a narrow range of values of these three parameters. To identify the optimum Hansen parameters for dispersing the as-prepared PGNFs, we plot in Fig. 6 the absorbance of the different suspensions as a function of $\delta_{D}, \delta_{P}$ and $\delta_{H}$, where we see that the dispersed amount is maximized roughly around 16-17 $\left(\delta_{D}\right), 4-6\left(\delta_{P}\right)$ and 14-16 $\left(\delta_{H}\right)$ $\mathrm{MPa}^{1 / 2}$. These values are fairly different to those previously reported for the successful solvents of carbon nanotubes and graphene [16,22-24]. Moreover, an estimate of the Hansen parameters for the as-prepared nanofibers, obtained from the weighted average of the solubility parameters of the solvents using the nanofiber absorbance for each dispersion as the weighting factor, yielded the following values: $\left\langle\delta_{D, N F}\right\rangle \approx 16.4 \pm 0.1$ $\mathrm{MPa}^{1 / 2},\left\langle\delta_{P, N F}\right\rangle \approx 8.6 \pm 0.6 \mathrm{MPa}^{1 / 2}$ and $\left\langle\delta_{H, N F}\right\rangle \approx 14.7 \pm 0.9 \mathrm{MPa}^{1 / 2}$. For comparison, the Hansen parameters of pristine graphene were evaluated by Hernandez et al. to be $\left\langle\delta_{D}\right) \approx$ 18.0 $\mathrm{MPa}^{1 / 2},\left\langle\delta_{P}\right) \approx 9.3 \mathrm{MPa}^{1 / 2}$ and $\left\langle\delta_{H}\right\rangle \approx 7.7 \mathrm{MPa}^{1 / 2}$ [16], with similar values having being reported also for carbon nanotubes. ${ }^{24}$ The most significant difference between the 
Hansen parameters of graphene/carbon nanotubes and those of the PGNFs deduced here lies in the hydrogen-bonding component, $\delta_{H}$, which is much larger for the latter. This result should come as no surprise considering that the surface of the as-prepared nanofibers is, as discussed above, heavily decorated with oxygen groups, many of which (hydroxyls, carboxyls, etc) can strongly interact through hydrogen bonding and other types of interaction (e.g., acid-base) described by the $\delta_{H}$ parameter. In the same line of reasoning, it is also not unexpected that many of the successful solvents of the as-prepared PGNFs are alcohols, which are readily prone to form hydrogen bonds. Furthermore, because high temperature annealing of the nanofibers changes their surface structure to a one dominated by (curved) basal planes (Fig. 1), the dispersion behavior of the HT-PGNFs should be expected to differ from that of their as-prepared counterparts, and more specifically, to be similar to the dispersibility of carbon nanotubes and graphene. Indeed, dispersion experiments carried out with a representative suite of solvents indicated that alcohols could no longer solubilize the nanofibers after the heat treatment, whereas typical successful solvents of nanotubes and graphene, such as $N$-methyl-2-pyrrolidone, were also effective for the HT-PGNFs. 

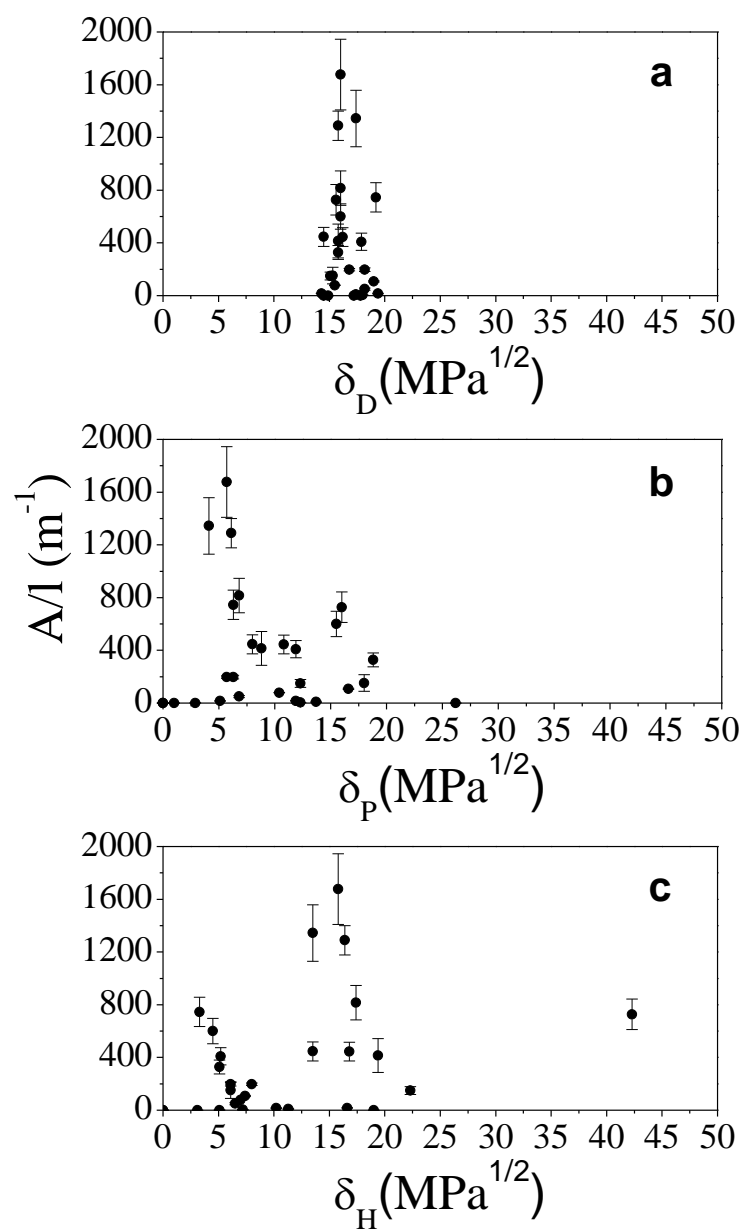

Fig. 6. Absorbance at $660 \mathrm{~nm}$ of PGNF dispersions in solvents plotted as a function of the dispersive (a), polar (b) and hydrogen-bonding (c) Hansen solubility parameters of the solvents.

To validate the previous Hansen solubility parameter analysis of the nanofibers, the absorbance measured for the different dispersions was plotted (Fig. 7) against the PGNF-solvent distance in the Hansen parameter space, $R$, given by the following expression [50]:

$$
R=\sqrt{4\left(\delta_{D, N F}-\delta_{D, s o l}\right)^{2}+\left(\delta_{P, N F}-\delta_{P, s o l}\right)^{2}+\left(\delta_{H, N F}-\delta_{H, s o l}\right)^{2}}
$$


If the Hansen parameters obtained for the nanofibers are correct (or at least, approximately correct), then the dispersed amount should tend to increase with decreasing $R$. Although with some scattering, such a trend is actually observed in Fig. 7, indicating that the dispersibility of this type of carbon material can be reasonably accounted for by means of the Hansen parameter theory.

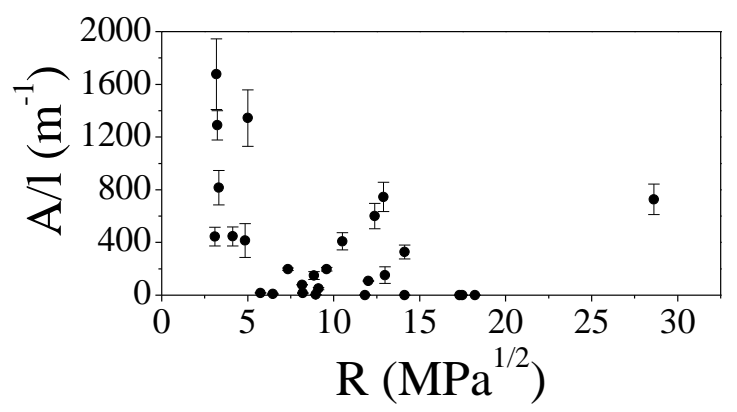

Fig. 7. Absorbance at $660 \mathrm{~nm}$ of PGNF dispersions in solvents plotted as a function of the distance, R, between PGNF and solvent in the Hansen parameter space.

The nanofiber dispersions were seen to be remarkably stable in almost all the identified good solvents: the amount of sediment developed over the course of weeks to months was very small or even undetectable to the naked eye. Water was the only notable exception to this rule. Although a relatively large quantity of nanofibers could be initially suspended in water using the reported procedure (see Table S1 in the ESI), most of it precipitated in a matter of just a few days. Fig. 8 shows the sedimentation behavior of nanofiber dispersions in the best solvent (1-butanol), a good solvent (ethanol) and a comparatively poor solvent ( $N$-cyclohexyl-2-pyrrolidone), obtained by following the suspension absorbance at $660 \mathrm{~nm}$ over time. While only $\sim 60 \%$ of the amount of material suspended immediately after the centrifugation step was still present in the latter solvent $550 \mathrm{~h}$ later, 1-butanol and ethanol were able to retain more than 
$95 \%$ of their original dispersed phase after a similar standing time. We believe that at least one of the reasons for the high stability in the best solvents is the fact that the nanofibers are suspended in the liquid phase mainly as individual, well-separated entities (Fig. 4c), rather than as entangled aggregates. FE-SEM imaging indicated that such aggregates (made up of a few to several nanofibers) were indeed noticeably present in the dispersions if the mild sonication step was omitted from their preparation procedure, and in this case a larger amount of sediment was observed. We also note that the typical dispersion procedure used here [stirring (350 rpm, $2 \mathrm{~h})$, sonication (30 min) and centrifugation $(3000 \mathrm{~g}, 5 \mathrm{~min})]$ afforded significant nanofiber concentrations in the most effective solvents. The concentrations were estimated by determining the weight of dispersions with known volume after vacuum filtration through a silver membrane filter (pore size $\sim 0.2 \mu \mathrm{m}$ ). For a starting nanofiber concentration of $5 \mathrm{mg} \mathrm{mL}^{-1}$, the dispersion procedure yielded concentrations after centrifugation of $\sim 0.6 \mathrm{mg} \mathrm{mL}^{-1}$ in 1 butanol, $\sim 0.3-0.5 \mathrm{mg} \mathrm{mL}^{-1}$ in isopropyl alcohol and cyclohexanol, or $\sim 0.1-0.3 \mathrm{mg} \mathrm{mL}^{-1}$ in ethanol, 1-propanol, allyl alcohol, o-dichlorobenzene, acetic acid, nitromethane, nitroethane and cyclopentanone. Such concentrations have not been optimized, but can be increased by changing some experimental parameters (e.g., larger starting nanofiber concentrations and/or longer stirring times).

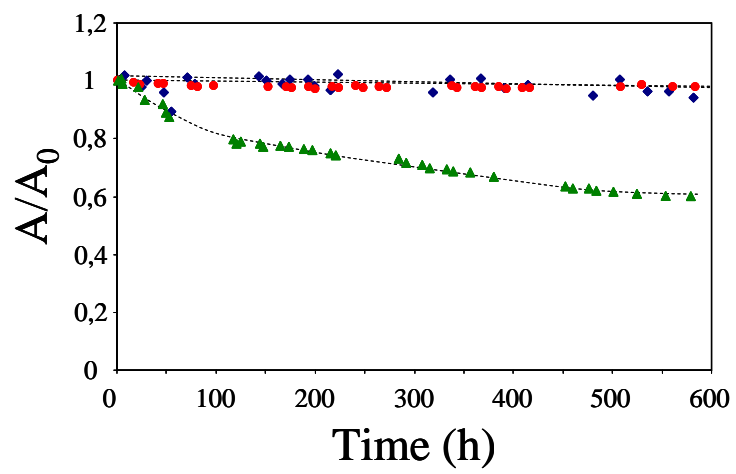


Fig. 8. Stability plots of PGNF dispersions in 1-butanol (blue diamonds), ethanol (red circles) and $N$-cyclo-hexyl-2-pyrrolidone (green triangles).

An additional point that should be commented on concerns the fact that the solutesolvent enthalpies of mixing described by equations (1) to (3) take explicit account of the energetic cost of breaking up the solute material into its individual entities (i.e., those that will be finally dispersed in the solvent). Inclusion of the appropriate term in the energy balance is clearly required as a rule, because the solute entities are in most cases cohesively interacting with each other before they are dispersed in the solvent. For instance, carbon nanotubes and graphene are typically dispersed in solvents by exfoliating them from nanotube bundles and graphite, respectively, which obviously involves an energy cost that has to be allowed for. On the other hand, the starting PGNF material investigated here is already in a rather loose, disaggregated state, and so an energy cost associated to overcoming cohesive interactions between the nanofibers does not probably come into play during the dispersion process. Consequently, incorporating the corresponding energy term in the enthalpies of mixing might not be completely justified in the present case. If we remove such a term from the surface energy-based analysis of the enthalpy of mixing, equation (1) can be re-written as

$$
\Delta H_{m i x}=\frac{4}{w}\left[\left(\delta_{N F}-\delta_{s o l}\right)^{2}-\delta_{N F}^{2}\right] \phi
$$

Because the only difference between equations (1) and (5) is the second term on the right-hand side of the latter, which is a constant for a given solute, both equations predict the same general dispersion behavior: the closer the surface energy of a solvent is to that of the PGNFs, the lower the energetic cost of the dispersion and so the larger the dispersed amount of nanofibers in that solvent should be. A similar reasoning can be applied to the analysis based on the Hildebrand and Hansen solubility parameters 
[equations (2) and (3)], leading to similar conclusions. Therefore, we believe that the relative ability of solvents to disperse the PGNFs (or, by the same token, other nanomaterials) does not depend on the aggregation state of the starting material.

\section{Conclusions}

An analysis based on surface energies and Hansen solubility parameters has been successfully carried out to rationalize the dispersion behavior of platelet-type graphite nanofibers (PGNFs) in solvents as well as to assist in the identification of good solvents for this type of carbon nanomaterial. The dispersion behavior of PGNFs was shown to be clearly different to that exhibited by pristine carbon nanotubes and graphene. This result can be mainly put down to the peculiar surface structure of the nanofibers, which is constituted by edge planes saturated with oxygen functional groups. By contrast, the surface of carbon nanotubes and graphene is dominated by basal planes. Successful solvents for the PGNFs are characterized by surface tensions typically between $\sim 25$ and $35 \mathrm{~mJ} \mathrm{~m}^{-2}$ (compared to $\sim 40 \mathrm{~mJ} \mathrm{~m}^{-2}$ for carbon nanotubes and graphene) and relatively large values of the hydrogen-bonding Hansen solubility parameter $\left(\delta_{\mathrm{H}} \sim 14-16 \mathrm{MPa}^{1 / 2}\right)$, thus including many alcohols such as 1-butanol, ethanol, allyl alcohol or cyclohexanol.

\section{Acknowledgements}

Financial support from the Spanish MINECO (projects MAT2008-05700 and MAT2011-26399) is gratefully acknowledged. L.G. thanks the receipt of a post-doctoral contract (JAE-Doc) from CSIC.

\section{References}


[1] Burda C, Chen X, Narayanan R, El-Sayed MA. Chemistry and properties of nanocrystals of different shapes. Chem Rev 2005; 105: 1025-102.

[2] Sau TK, Rogach AL, Jackel F, Klar TA, Feldmann J. Properties and applications of colloidal nonspherical noble metal nanoparticles. Adv Mater 2010; 22: 1805-25.

[3] Vaisman L, Wagner HD, Marom G. The role of surfactants in dispersion of carbon nanotubes. Adv Colloid Interface Sci 2006; 128-130: 37-46.

[4] Coleman JN. Liquid-Phase Exfoliation of Nanotubes and Graphene. Adv Funct Mater 2009; 19: 3680-95.

[5] Kim SW, Kim T, Kim YS, Choi HS, Lim HJ, Yang SJ, et al. Surface modifications for the effective dispersion of carbon nanotubes in solvents and polymers. Carbon 2012; 50: $3-33$.

[6] Premkumar T, Mezzenga R, Geckeler KE. Carbon nanotubes in the liquid phase: addressing the issue of dispersion. Small 2012; 8: 1299-313.

[7] Green AA, Hersam MC. Emerging methods for producing monodisperse graphene dispersions. J Phys Chem Lett 2010; 1: 544-9.

[8] Cui X, Zhang C, Hao R, Hou Y. Liquid-phase exfoliation, functionalization and applications of graphene. Nanoscale 2011; 3: 2118-26.

[9] Schnorr JM, Swager TM. Emerging applications of carbon nanotubes. Chem Mater 2011; 23: 646-57.

[10] Bai H, Li C, Shi G. Functional composite materials based on chemically converted graphene. Adv Mater 2011; 23: 1089-115.

[11] Lotya M, Hernandez Y, King PJ, Smith RJ, Nicolosi V, Karlsson LS, et al. Liquid phase production of graphene by exfoliation of graphite in surfactant/water solutions. J Am Chem Soc 2009; 131: 3611-20. 
[12] Guardia L, Fernández-Merino MJ, Paredes JI, Solís-Fernández P, Villar-Rodil S, Martínez-Alonso A, et al. High-throughput production of pristine graphene in an aqueous dispersion assisted by non-ionic surfactants. Carbon 2011; 49: 1653-62.

[13] Seo J-WT, Green AA, Antaris AL, Hersam MC. High-concentration aqueous dispersions of graphene using nonionic, biocompatible block copolymers. J Phys Chem Lett 2011; 2: 1004-8.

[14] Wang Y, Di C, Liu Y, Kajiura H, Ye S, Cao L, et al. Optimizing single-walled carbon nanotube films for applications in electroluminescent devices. Adv Mater 2008; 20: 4442-9.

[15] Hernandez Y, Nicolosi V, Lotya M, Blighe FM, Sun Z, De S, et al. High-yield production of graphene by liquid-phase exfoliation of graphite. Nature Nanotech 2008; 3: 563-568.

[16] Hernandez Y, Lotya M, Rickard D, Bergin SD, Coleman JN. Measurement of multicomponent solubility parameters for graphene facilitates solvent discovery. Langmuir, 2010; 26: 3208-13.

[17] Paredes JI, Villar-Rodil S, Martínez-Alonso A, Tascón JMD. Graphene oxide dispersions in organic solvents. Langmuir, 2008; 24: 10560-4.

[18] Park S, An J, Jung I, Piner RD, An SJ, Li X, et al. Colloidal suspensions of highly reduced graphene oxide in a wide variety of organic solvents. Nano Lett 2009; 9: 15937.

[19] Choi E-Y, Han TH, Hong J, Kim JE, Lee SH, Kim HW, et al. Noncovalent functionalization of graphene with end-functional polymers. J Mater Chem 2010; 20: 1907-12. 
[20] Ausman KD, Piner R, Lourie O, Ruoff RS. Organic solvent dispersions of singlewalled carbon nanotubes: Toward solutions of pristine nanotubes. J Phys Chem B 2000; 104: 8911-5.

[21] Landi BJ, Ruf HJ, Worman JJ, Raffaelle RP. Effects of alkyl amide solvents on the dispersion of single-wall carbon nanotubes. J Phys Chem B 2004; 108: 17089-95.

[22] Ham HT, Choi YS, Chung IJ. An explanation of dispersion states of single-walled carbon nanotubes in solvents and aqueous surfactant solutions using solubility parameters. J Colloid Interface Sci 2005; 286: 216-23.

[23] Detriche S, Zorzini G, Colomer JF, Fonseca A, Nagy JB. Application of the Hansen solubility parameters theory to carbon nanotubes. J Nanosci Nanotechnol 2008; 8: 6082-92.

[24] Bergin SD, Sun Z, Rickard D, Streich PV, Hamilton JP, Coleman JN. Multicomponent solubility parameters for single-walled carbon nanotube-solvent mixtures. ACS Nano 2009; 3: 2340-50.

[25] Pierson HO. Handbook of carbon, graphite, diamond and fullerenes. Park Ridge NJ: Noyes. 1993.

[26] Subramoney S. Novel nanocarbons-structure, properties, and potential applications. Adv. Mater 1998; 10: 1157-71.

[27] Jian K, Yan A, Külaots I, Crawford GP, Hurt RH. Reconstruction and hydrophobicity of nanocarbon surfaces composed solely of graphene edges. Carbon 2006; 44: 2102-6.

[28] Rodriguez NM, Chambers A, Baker RTK. Catalytic engineering of carbon nanostructures. Langmuir 1995; 11: 3862-66. 
[29] De Jong KP, Geus JW. Carbon nanofibers : Catalytic synthesis and applications. Catal Rev-Sci Eng $2000 ; 42: 481-510$.

[30] Chen X, Xu Z-H, Li X, Shaibat MA, Ishii Y, Ruoff RS. Structural and mechanical characterization of platelet graphite nanofibers. Carbon, 2007 ; 45: 416-23.

[31] Serp P, Corrias M, Kalck P. Carbon nanotubes and nanofibers in catalysis. Appl Catal A: Gen $2003 ; 253: 337-58$.

[32] Tsuji M, Kubokawa M, Yano R, Miyamae N, Tsuji T, Jun M-S, et al. Fast preparation of PtRu catalysts supported on carbon nanofibers by the microwave-polyol method and their application to fuel cells. Langmuir $2007 ; 23: 387-90$.

[33] Takahashi M, Motoyama Y, Higashi K, Yoon S-H, Mochida I, Nagashima H. Chemoselective hydrogenation of nitroarenes with carbon nanofiber-supported platinum and palladium nanoparticles. Org Lett $2008 ; 10: 1601-4$.

[34] Qin Y-H, Yang H-H, Zhang X-S, Li P, Ma C-A. Effect of carbon nanofibers microstructure on electrocatalytic activities of Pd electrocatalysts for ethanol oxidation in alkaline medium. Int J Hydrog Energy 2010; 35: 7667-74.

[35] Oh H-S, Kim H. Efficient synthesis of Pt nanoparticles supported on hydrophobic graphitized carbon nanofibers for electrocatalysts using noncovalent functionalization. Adv. Funct. Mater 2011; 21: 3954-60.

[36] Ambrosi A, Sasaki T, Pumera M. Platelet graphite nanofibers for electrochemical sensing and biosensing: The influence of graphene sheet orientation. Chem Asian J 2010; 5: 266-71.

[37] Kim S, Kim B, Jeong H-G, Rhee CK, Lim T-H. Electrochemical capacitance of electrochemically oxidized $\mathrm{Ru}$ nanoparticles of various sizes on platelet carbon nanofiber. Bull Korean Chem Soc 2010; 31: 3852-5. 
[38] Lim S, Yoon S-H, Mochida I, Chi, J-H. Surface modification of carbon nanofiber with high degree of graphitization. J. Phys. Chem. B 2004; 108: 1533-6.

[39] Gogotsi Y, Libera JA, Kalashnikov N, Yoshimura M. Graphite polyhedral crystals. Science 2000; 290: 317-20.

[40] Kim T, Lim S, Kwon K, Hong S-H, Qiao W, Rhee CK, et al. Electrochemical capacitances of well-defined carbon surfaces. Langmuir, 2006; 22: 9086-8.

[41] Campos-Delgado J, Kim YA, Hayashi T, Morelos-Gómez A, Hofmann M, Muramatsu H, et al. Thermal stability studies of CVD-grown graphene nanoribbons: Defect annealing and loop formation. Chem Phys Lett 2009; 469: 177-82.

[42] Long D, Li W, Qiao W, Miyawaki J, Yoon S-H, Mochida I, et al. Graphitization behaviour of chemically derived graphene sheets. Nanoscale 2011; 3: 3652-6.

[43] Chang H, Bard AJ. Scanning tunneling microscopy studies of carbon-oxygen reactions on highly oriented pyrolytic graphite. J Am Chem Soc 1991; 113: 5588-96.

[44] Hahn JR. Kinetic study of graphite oxidation along two lattice directions. Carbon 2005; 43: 1506-11.

[45] Ferrari AC, Robertson J. Interpretation of Raman spectra of disordered and amorphous carbon. Phys Rev B 2000; 61: 14095-107.

[46] Pimenta MA, Dresselhaus G, Dresselhaus MS, Cançado LG, Jorio A, Saito R. Studying disorder in graphite-based systems by Raman spectroscopy. Phys Chem Chem Phys 2007; 9 : 1276-91.

[47] Leon y Leon CA, Radovic LR. Interfacial chemistry and electrochemistry of carbon surfaces. Chem. Phys. Carbon 1994; 24: 213-310.

[48] Boehm HP. Surface oxides on carbon and their analysis: a critical assessment. Carbon $2002 ; 40: 145-9$. 
[49] Rosenthal D, Ruta M, Schlögl R, Kiwi-Minsker L. Combined XPS and TPD study of oxygen-functionalized carbon nanofibers grown on sintered metal fibers. Carbon 2010; 48: 1835-43.

[50] Hansen CM. Hansen Solubility Parameters: A User’s Handbook. Boca Raton: CRC Press; 2007.

[51] Coleman JN, Lotya M, O’Neill A, Bergin SD, King PJ, Khan U. Two-dimensional nanosheets produced by liquid exfoliation of layered materials. Science 2011; 331: 56871.

[52] Zhou K-G, Mao N-N, Wang H-X, Peng Y, Zhang H-L. A mixed-solvent strategy for efficient exfoliation of inorganic graphene analogues. Angew Chem Int Ed. 2011; 50: $10839-42$.

[53] Jiang L, Gao L, Sun J. Production of aqueous colloidal dispersions of carbon nanotubes. J Colloid Interface Sci 2003; 260: 89-94.

[54] Li D, Müller MB, Gilje S, Kaner RB, Wallace GG. Processable aqueous dispersions of graphene nanosheets. Nature Nanotech 2008; 3: 101-5.

[55] Riddick JA, Bunger WB, Sakano TK. Organic Solvents: Physical Properties and Methods of Purification. New York: John Wiley \& Sons; 1986.

[56] Abrahamson J. The surface energies of graphite. Carbon 1973; 11: 337-62.

[57] Donnet JB, Brendle M, Dhami TL, Bahl OP. Plasma treatment effect on the surface energy of carbon and carbon fibers. Carbon 1986; 24: 757-70. 


\section{Figure captions}

Fig. 1. Representative FE-SEM images of the as-prepared (a) and heat-treated (b) PGNFs. TEM images of the as-prepared (c) and heat-treated (d) nanofibers. Schematic representations of the PGNF surface structure are given as insets to (c) and (d).

Fig. 2. Thermogravimetric (TG, panel a) and differential thermogravimetric (DTG, panel b) plots of the PGNFs (black) and HT-PGNFs (gray) and of a natural graphite powder (red). First-order Raman spectra (c) and XRD patterns (d) for the PGNFS (black) and HT-PGNFs (gray).

Fig. 3. X-ray photoelectron spectra recorded for the PGNFs (black) and HT-PGNFs (gray): survey spectra (a), and high resolution core level spectra for C1s (b) and O1s (c).

Fig. 4. (a) Digital photographs of cuvettes containing dispersions of as-prepared PGNFs in the following solvents (the bracketed numbers refer to their surface tension in $\mathrm{mJ} \mathrm{m}^{-}$ $\left.{ }^{2}\right)$. A: $n$-pentane (16.05); B: $n$-hexane (18.42); C: ethanol (22.32); D: 1-butanol (24.67); E: $o$-dichlorobenzene (26.84); F: acetonitrile (29.29); G: cyclopentanone (33.87); H: $N, N$-dimethylformamide (36.76); I: $N$-cyclohexyl-2-pyrrolidone (38.8); J: $N$-methyl-2pyrrolidone (40.07). (b) UV-vis absorption spectrum of PGNF dispersion in 1-butanol. (c) FE-SEM image of a typical PGNF dispersion drop-cast onto HOPG.

Fig. 5. Absorbance at $660 \mathrm{~nm}$ of PGNF dispersions in solvents plotted as a function of solvent surface tension (a) and Hildebrand solubility parameter (b). 
Fig. 6. Absorbance at $660 \mathrm{~nm}$ of PGNF dispersions in solvents plotted as a function of the dispersive (a), polar (b) and hydrogen-bonding (c) Hansen solubility parameters of the solvents.

Fig. 7. Absorbance at $660 \mathrm{~nm}$ of PGNF dispersions in solvents plotted as a function of the distance, $\mathrm{R}$, between PGNF and solvent in the Hansen parameter space.

Fig. 8. Stability plots of PGNF dispersions in 1-butanol (blue diamonds), ethanol (red circles) and $N$-cyclo-hexyl-2-pyrrolidone (green triangles). 


\section{Table captions}

Table 1. Comparison of rate constants normalized to the area $\left(\mathrm{k}_{1}\right)$ or mass $\left(\mathrm{k}_{2}\right)$ per unit volume of reaction for a number of catalysts based on silver nanoparticles (Ag NPs) reported in the literature. 